\title{
Electron localizability indicator for correlated wavefunctions. III: singlet and triplet pairs
}

\author{
M. Kohout · F. R. Wagner · Yu. Grin
}

Received: 25 June 2007 / Accepted: 22 October 2007 / Published online: 14 November 2007

(C) Springer-Verlag 2007

\begin{abstract}
The electron pair density can be decomposed into the symmetric and antisymmetric parts. The antisymmetric component is connected with the probability that two electrons are coupled to a triplet. On the basis of triplet-coupled electrons the electron localizability indicator is defined, describing the correlation of motion of electrons forming a triplet pair. In case of spin-polarized systems the electron localizability indicator for triplet pairs combines the two spin channels together into a single functional.
\end{abstract}

Keywords Electron localizability indicator .

Triplet-coupled electrons

\section{Introduction}

The space partitioning based on the division of the whole space into compact space filling mutually exclusive regions (micro-cells) enclosing a fixed amount of same-spin electron pairs (the $\omega$-restricted partitioning) leads the electron localizability indicator (ELI) given as the distribution of charges needed to form a same-spin electron pair (the $\omega$-restricted populations), cf. Ref. [1] denoted in the following as Part I. In case of high mutual avoidance of same-spin electrons high average number of electrons is needed to form the fixed fraction of same-spin pair in the micro-cell. From this point of view ELI describes the correlation of electronic motion of same-spin electrons [2]. ELI was used to detect signatures of chemical bonding in molecules.

M. Kohout $(\varangle)$ · F. R. Wagner · Yu. Grin

Max-Planck-Institut für Chemische Physik fester Stoffe,

Nöthnitzer Str. 40, 01187 Dresden,

Germany

e-mail: kohout@cpfs.mpg.de
Similar procedure, based on integrals of opposite-spin electron pair density over micro-cells of $\omega$-restricted partitioning using fixed amount of charge, leads to the electron localizability indicator for antiparallel-spin pairs (ELIA), cf. Ref. [3] denoted in the following as Part II. In case of high mutual avoidance of opposite-spin electrons less opposite-spin pairs are found in the micro-cell. For microcells restricted to enclose a fixed product of $\alpha$-spin and $\beta$-spin charge the number of opposite-spin pairs in the respective micro-cells is a non-constant distribution only for explicitly correlated wavefunctions. For correlated wavefunction it was shown that ELIA signatures can be used to characterize the bonding situation from the viewpoint of opposite-spin pairs.

In case of spin-polarized systems two different ELI distributions will be obtained for the above mentioned partitioning scheme, one for each spin channel. The widely used electron localization function (ELF) [4], which in certain sense can be seen as an approximation to ELI, encountered similar situation (the derivation of ELF by Becke and Edgecombe was not restricted to the closed-shell systems only, although all their examples were). With the interpretation of ELF based on the kinetic energy densities [5], it was proposed to use for spin-polarized systems an ELF formula with the kinetic energy densities split into spin-dependent parts [6], resulting in a single "spin-polarized" ELF formula (using the total kinetic energy densities without this spin-dependent splitting yields the "closed-shell" formula). For ELI, which of course is not based on kinetic energy densities, we propose another way to formulate a single functional for the spin-polarized case. This approach, described in following sections, is based on the decomposition of the electron pair density into the symmetric and antisymmetric parts. Usage of the triplet pair density (the antisymmetric part) enables to formulate a single indicator including both spin parts in a way consistent with the approach of restricted populations. Additionally, it 
will be shown that ELIA, which has no equivalent in ELF, is connected with the singlet pair density (the symmetric part of the decomposition).

\section{Theory}

The same-spin electron pair density originates from the decomposition of the electron pair density into same-spin and opposite-spin parts. Though, this decomposition is not invariant with respect to the rotation in the spin space $[7,8]$. The spinless two-particle density matrix $\rho_{2}\left(\mathbf{r}_{1}^{\prime} \mathbf{r}_{2}^{\prime}, \mathbf{r}_{1} \mathbf{r}_{2}\right)$ for the $N$-electron wavefunction $\Psi\left(\mathbf{x}_{1} \mathbf{x}_{2} \cdots \mathbf{x}_{N}\right)[9,10]$ :

$$
\begin{aligned}
& \rho_{2}\left(\mathbf{r}_{1}^{\prime} \mathbf{r}_{2}^{\prime}, \mathbf{r}_{1} \mathbf{r}_{2}\right)=\left(\begin{array}{c}
N \\
2
\end{array}\right) \int \mathrm{d} \sigma_{1} \int \mathrm{d} \sigma_{2} \int \mathrm{d} \mathbf{x}_{3} \cdots \\
& \times \int \mathrm{d} \mathbf{x}_{N} \Psi\left(\mathbf{x}_{1}^{\prime} \mathbf{x}_{2}^{\prime} \cdots \mathbf{x}_{N}^{\prime}\right) \Psi^{*}\left(\mathbf{x}_{1} \mathbf{x}_{2} \cdots \mathbf{x}_{N}\right),
\end{aligned}
$$

with the combined position and spin coordinates $\mathbf{x}_{i}=\left(\mathbf{r}_{i}\right.$, $\sigma_{i}$ ), can always be decomposed into the symmetric and antisymmetric parts $\rho_{2}^{(s)}\left(\mathbf{r}_{1}^{\prime} \mathbf{r}_{2}^{\prime}, \mathbf{r}_{1} \mathbf{r}_{2}\right)$ and $\rho_{2}^{(t)}\left(\mathbf{r}_{1}^{\prime} \mathbf{r}_{2}^{\prime}, \mathbf{r}_{1} \mathbf{r}_{2}\right)$, respectively $[11,12]$ :

$\rho_{2}\left(\mathbf{r}_{1}^{\prime} \mathbf{r}_{2}^{\prime}, \mathbf{r}_{1} \mathbf{r}_{2}\right)=\rho_{2}^{(s)}\left(\mathbf{r}_{1}^{\prime} \mathbf{r}_{2}^{\prime}, \mathbf{r}_{1} \mathbf{r}_{2}\right)+\rho_{2}^{(t)}\left(\mathbf{r}_{1}^{\prime} \mathbf{r}_{2}^{\prime}, \mathbf{r}_{1} \mathbf{r}_{2}\right)$,

with

$$
\begin{aligned}
\rho_{2}^{(s)}\left(\mathbf{r}_{1}^{\prime} \mathbf{r}_{2}^{\prime}, \mathbf{r}_{1} \mathbf{r}_{2}\right)= & \frac{1}{2}\left[\rho_{2}\left(\mathbf{r}_{1}^{\prime} \mathbf{r}_{2}^{\prime}, \mathbf{r}_{1} \mathbf{r}_{2}\right)\right. \\
& \left.+\rho_{2}\left(\mathbf{r}_{1}^{\prime} \mathbf{r}_{2}^{\prime}, \mathbf{r}_{2} \mathbf{r}_{1}\right)\right] \\
\rho_{2}^{(t)}\left(\mathbf{r}_{1}^{\prime} \mathbf{r}_{2}^{\prime}, \mathbf{r}_{1} \mathbf{r}_{2}\right)= & \frac{1}{2}\left[\rho_{2}\left(\mathbf{r}_{1}^{\prime} \mathbf{r}_{2}^{\prime}, \mathbf{r}_{1} \mathbf{r}_{2}\right)\right. \\
& \left.-\rho_{2}\left(\mathbf{r}_{1}^{\prime} \mathbf{r}_{2}^{\prime}, \mathbf{r}_{2} \mathbf{r}_{1}\right)\right] .
\end{aligned}
$$

The diagonal elements of the above matrices are the singlet and triplet pair densities $\rho_{2}^{(s)}\left(\mathbf{r}_{1}, \mathbf{r}_{2}\right)$ and $\rho_{2}^{(t)}\left(\mathbf{r}_{1}, \mathbf{r}_{2}\right)$, respectively, with integrals proportional to the probability that two electrons are coupled to a singlet, resp. a triplet. The total numbers $D^{(s)}$ and $D^{(t)}$ of singlet resp. triplet electron pairs equal (with the normalization of Eq. 1) [11]:

$$
\begin{aligned}
& D^{(s)}=\frac{1}{8} N(N+2)-\frac{1}{2} S(S+1) \\
& D^{(t)}=\frac{3}{8} N(N-2)+\frac{1}{2} S(S+1) .
\end{aligned}
$$

The singlet and triplet electron densities $\rho^{(s)}(\mathbf{r})$ and $\rho^{(t)}(\mathbf{r})$, respectively, are given by:

$$
\begin{aligned}
& \rho^{(s)}(\mathbf{r})=\frac{1}{4} \frac{N+2}{N-1} \rho(\mathbf{r})-\frac{1}{2} \frac{S+1}{N-1} \rho_{S}(\mathbf{r}) \\
& \rho^{(t)}(\mathbf{r})=\frac{3}{4} \frac{N-2}{N-1} \rho(\mathbf{r})+\frac{1}{2} \frac{S+1}{N-1} \rho_{S}(\mathbf{r}),
\end{aligned}
$$

with with total spin quantum number $S$ and the spin density $\rho_{s}(\mathbf{r})=\rho_{\alpha}(\mathbf{r})-\rho_{\beta}(\mathbf{r})$. The singlet and triplet electron densities integrate to the total number of electrons $N^{(s)}$ and $N^{(t)}$ that couple to a singlet and triplet, respectively:

$$
\begin{aligned}
& N^{(s)}=\frac{\frac{1}{4} N(N+2)-S(S+1)}{N-1} \\
& N^{(t)}=\frac{\frac{3}{4} N(N-2)+S(S+1)}{N-1} .
\end{aligned}
$$

To derive the expression for the electron localizability indicators for the singlet and triplet-coupled electrons let us write the reduced 2-matrix in familiar terms, i.e., using the samespin and opposite-spin 2-matrices:

$$
\begin{aligned}
\rho_{2}\left(\mathbf{r}_{1}^{\prime} \mathbf{r}_{2}^{\prime}, \mathbf{r}_{1} \mathbf{r}_{2}\right)= & \rho_{2}^{\alpha \alpha}\left(\mathbf{r}_{1}^{\prime} \mathbf{r}_{2}^{\prime}, \mathbf{r}_{1} \mathbf{r}_{2}\right)+\rho_{2}^{\beta \beta}\left(\mathbf{r}_{1}^{\prime} \mathbf{r}_{2}^{\prime}, \mathbf{r}_{1} \mathbf{r}_{2}\right) \\
& +\rho_{2}^{\alpha \beta}\left(\mathbf{r}_{1}^{\prime} \mathbf{r}_{2}^{\prime}, \mathbf{r}_{1} \mathbf{r}_{2}\right)+\rho_{2}^{\beta \alpha}\left(\mathbf{r}_{1}^{\prime} \mathbf{r}_{2}^{\prime}, \mathbf{r}_{1} \mathbf{r}_{2}\right) .
\end{aligned}
$$

The same-spin 2-matrices for a configuration interaction (CI) wavefunction constructed from normalized Slater determinants using $n$ orthonormal orbitals $\phi$ read (cf. Part I):

$$
\begin{aligned}
& \rho_{2}^{\alpha \alpha}\left(\mathbf{r}_{1}^{\prime} \mathbf{r}_{2}^{\prime}, \mathbf{r}_{1} \mathbf{r}_{2}\right) \\
& =\frac{1}{2} \sum_{i<j}^{n} \sum_{k<l}^{n} P_{i j, k l}^{\alpha \alpha}\left|\phi_{i}\left(\mathbf{r}_{1}^{\prime}\right) \phi_{j}\left(\mathbf{r}_{2}^{\prime}\right)\right|\left|\phi_{k}^{*}\left(\mathbf{r}_{1}\right) \phi_{l}^{*}\left(\mathbf{r}_{2}\right)\right| \\
& \rho_{2}^{\beta \beta}\left(\mathbf{r}_{1}^{\prime} \mathbf{r}_{2}^{\prime}, \mathbf{r}_{1} \mathbf{r}_{2}\right) \\
& =\frac{1}{2} \sum_{i<j}^{n} \sum_{k<l}^{n} P_{i j, k l}^{\beta \beta}\left|\phi_{i}\left(\mathbf{r}_{1}^{\prime}\right) \phi_{j}\left(\mathbf{r}_{2}^{\prime}\right)\right|\left|\phi_{k}^{*}\left(\mathbf{r}_{1}\right) \phi_{l}^{*}\left(\mathbf{r}_{2}\right)\right|,
\end{aligned}
$$

with the discrete representations $P_{i j, k l}^{\alpha \alpha}$ and $P_{i j, k l}^{\beta \beta}$ for the samespin combinations of the orbitals. Two Slater determinants differing at most by same-spin orbitals $i, j$ and $k, l$, respectively, contribute to the element $[i j, k l]$. One is free to ascribe this contribution to the element $[j i, k l]$ instead (of course, with opposite sign). But it is more convenient to accumulate all such contributions in the matrix elements where $i<j$ and $k<l$.

The opposite-spin 2-matrices for a CI wavefunction are given by (cf. Part II):

$$
\begin{aligned}
& \rho_{2}^{\alpha \beta}\left(\mathbf{r}_{1}^{\prime} \mathbf{r}_{2}^{\prime}, \mathbf{r}_{1} \mathbf{r}_{2}\right) \\
& =\frac{1}{2} \sum_{i, k}^{n} \sum_{j, l}^{n} P_{i j, k l}^{\alpha \beta} \phi_{i}\left(\mathbf{r}_{1}^{\prime}\right) \phi_{j}\left(\mathbf{r}_{2}^{\prime}\right) \phi_{k}^{*}\left(\mathbf{r}_{1}\right) \phi_{l}^{*}\left(\mathbf{r}_{2}\right) \\
& \rho_{2}^{\beta \alpha}\left(\mathbf{r}_{1}^{\prime} \mathbf{r}_{2}^{\prime}, \mathbf{r}_{1} \mathbf{r}_{2}\right) \\
& =\frac{1}{2} \sum_{i, k}^{n} \sum_{j, l}^{n} P_{j i, l k}^{\beta \alpha} \phi_{j}\left(\mathbf{r}_{1}^{\prime}\right) \phi_{i}\left(\mathbf{r}_{2}^{\prime}\right) \phi_{l}^{*}\left(\mathbf{r}_{1}\right) \phi_{k}^{*}\left(\mathbf{r}_{2}\right),
\end{aligned}
$$

whereby

$$
P_{i j, k l}^{\alpha \beta}=P_{j i, l k}^{\beta \alpha}
$$

The simple product form for the opposite-spin terms results from the integration over spin.

From Eqs. 8 and 9 it can be seen that the exchange of the coordinates $\mathbf{r}_{1}$ and $\mathbf{r}_{2}$ yields for the same-spin 2-matrices:

$\rho_{2}^{\sigma \sigma}\left(\mathbf{r}_{1}^{\prime} \mathbf{r}_{2}^{\prime}, \mathbf{r}_{1} \mathbf{r}_{2}\right)=-\rho_{2}^{\sigma \sigma}\left(\mathbf{r}_{1}^{\prime} \mathbf{r}_{2}^{\prime}, \mathbf{r}_{2} \mathbf{r}_{1}\right)$,

whereas for the the opposite-spin 2-matrices, cf. Eqs. 10 and 11, the following applies:

$\rho_{2}^{\alpha \beta}\left(\mathbf{r}_{1}^{\prime} \mathbf{r}_{2}^{\prime}, \mathbf{r}_{1} \mathbf{r}_{2}\right)=\rho_{2}^{\alpha \beta}\left(\mathbf{r}_{1}^{\prime} \mathbf{r}_{2}^{\prime}, \mathbf{r}_{2} \mathbf{r}_{1}\right)$ for $\mathbf{r}_{1}=\mathbf{r}_{2}$ 
and

$\rho_{2}^{\alpha \beta}\left(\mathbf{r}_{1}^{\prime} \mathbf{r}_{2}^{\prime}, \mathbf{r}_{1} \mathbf{r}_{2}\right) \neq \rho_{2}^{\alpha \beta}\left(\mathbf{r}_{1}^{\prime} \mathbf{r}_{2}^{\prime}, \mathbf{r}_{2} \mathbf{r}_{1}\right)$ for $\mathbf{r}_{1} \neq \mathbf{r}_{2}$

Thus, the same-spin 2-matrices do not participate on the singlet pair density, cf. Eq. 3. Utilizing Eqs. 10-12 it follows that the singlet 2-matrix for CI wavefunction can be expressed as:

$$
\begin{aligned}
& \rho_{2}^{(s)}\left(\mathbf{r}_{1}^{\prime} \mathbf{r}_{2}^{\prime}, \mathbf{r}_{1} \mathbf{r}_{2}\right) \\
& =\frac{1}{4} \sum_{i, k}^{n} \sum_{j, l}^{n} P_{i j, k l}^{\alpha \beta}\left\|\phi_{i}\left(\mathbf{r}_{1}^{\prime}\right) \phi_{j}\left(\mathbf{r}_{2}^{\prime}\right)\right\|\left\|\phi_{k}^{*}\left(\mathbf{r}_{1}\right) \phi_{l}^{*}\left(\mathbf{r}_{2}\right)\right\|,
\end{aligned}
$$

with the permanents:

$\left\|\phi_{i}\left(\mathbf{r}_{1}^{\prime}\right) \phi_{j}\left(\mathbf{r}_{2}^{\prime}\right)\right\|=\phi_{i}\left(\mathbf{r}_{1}^{\prime}\right) \phi_{j}\left(\mathbf{r}_{2}^{\prime}\right)+\phi_{j}\left(\mathbf{r}_{1}^{\prime}\right) \phi_{i}\left(\mathbf{r}_{2}^{\prime}\right)$.

The permanent of a matrix is similar to the determinant but without taking into acount the signatures of the permutations, i.e., it represents the symmetric sum of the permutations.

The opposite-spin 2-matrices participate also on the triplet 2-matrix. However, due to the subtraction, cf. Eq. 3, the triplet 2-matrix involves determinants instead of the permanents. From Eq. 13 it is clear that the same-spin contributions to the triplet 2-matrix are directly given by the same-spin matrices of Eqs. 8 and 9. Thus, the triplet 2-matrix for a CI wavefunction reads:

$$
\begin{aligned}
& \rho_{2}^{(t)}\left(\mathbf{r}_{1}^{\prime} \mathbf{r}_{2}^{\prime}, \mathbf{r}_{1} \mathbf{r}_{2}\right)=\frac{1}{2} \sum_{i<j}^{n} \sum_{k<l}^{n}\left(P_{i j, k l}^{\alpha \alpha}\right. \\
& \left.\quad+P_{i j, k l}^{\beta \beta}\right)\left|\phi_{i}\left(\mathbf{r}_{1}^{\prime}\right) \phi_{j}\left(\mathbf{r}_{2}^{\prime}\right)\right|\left|\phi_{k}^{*}\left(\mathbf{r}_{1}\right) \phi_{l}^{*}\left(\mathbf{r}_{2}\right)\right| \\
& \quad+\frac{1}{4} \sum_{i, k}^{n} \sum_{j, l}^{n} P_{i j, k l}^{\alpha \beta}\left|\phi_{i}\left(\mathbf{r}_{1}^{\prime}\right) \phi_{j}\left(\mathbf{r}_{2}^{\prime}\right)\right|\left|\phi_{k}^{*}\left(\mathbf{r}_{1}\right) \phi_{l}^{*}\left(\mathbf{r}_{2}\right)\right| .
\end{aligned}
$$

Formally, the sum over the opposite-spin terms can be further simplified, because for all terms corresponding to $i=j$, respectively $k=l$, the determinants will vanish. Furthermore, the opposite-spin terms corresponding to the given combination of indexes $[i, j, k, l]$ can be accumulated into the single term $P_{i j, k l}^{o s}$ :

$P_{i j, k l}^{o s}=\frac{1}{2}\left(P_{i j, k l}^{\alpha \beta}-P_{i j, l k}^{\alpha \beta}+P_{j i, l k}^{\alpha \beta}-P_{j i, k l}^{\alpha \beta}\right)$,

with the superscript $o s$ for the opposite-spin contribution. Then, the triplet 2-matrix can be written as:

$$
\begin{aligned}
& \rho_{2}^{(t)}\left(\mathbf{r}_{1}^{\prime} \mathbf{r}_{2}^{\prime}, \mathbf{r}_{1} \mathbf{r}_{2}\right) \\
& =\frac{1}{2} \sum_{i<j}^{n} \sum_{k<l}^{n} P_{i j, k l}^{(t)}\left|\phi_{i}\left(\mathbf{r}_{1}^{\prime}\right) \phi_{j}\left(\mathbf{r}_{2}^{\prime}\right)\right|\left|\phi_{k}^{*}\left(\mathbf{r}_{1}\right) \phi_{l}^{*}\left(\mathbf{r}_{2}\right)\right|,
\end{aligned}
$$

with

$P_{i j, k l}^{(t)}=P_{i j, k l}^{\alpha \alpha}+P_{i j, k l}^{\beta \beta}+P_{i j, k l}^{o s}$.

\subsection{ELI for triplet-coupled electrons}

In Part I ELI was based on the approach of $q$-restricted space partitioning in order to achieve certain similarity with the ELF formula of Becke and Edgecombe. Later it was found to be more convenient to use ELI based on the approach of $D$-restricted space partitioning [13,14]. Following this idea the space is partitioned into compact space filling mutually exclusive micro-cells restricted to enclose a fixed fraction of an electron pair. Then, in each micro-cell the charge is determined. ELI is proportional to the distribution of such charges for infinitesimally small restriction. Focusing on triplet coupled electron pairs, now the micro-cells are restricted to enclose a fixed fraction of a triplet pair. Using the Taylor expansion of the triplet pair density the number of triplet pairs $D_{\mu}^{(t)}$ in the micro-cell $\mu$ centred around the position $\mathbf{a}_{\mu}$ is approximately given by (cf. Part I):

$D_{\mu}^{(t)} \approx \frac{1}{12} V_{\mu}^{8 / 3} g^{(t)}\left(\mathbf{a}_{\mu}\right)$,

where

$$
\begin{aligned}
g^{(t)}\left(\mathbf{a}_{\mu}\right)= & \sum_{i<j}^{n} \sum_{k<l}^{n} P_{i j, k l}^{(t)} \\
& \times\left[\phi_{i}\left(\mathbf{a}_{\mu}\right) \nabla \phi_{j}\left(\mathbf{a}_{\mu}\right)-\phi_{j}\left(\mathbf{a}_{\mu}\right) \nabla \phi_{i}\left(\mathbf{a}_{\mu}\right)\right] \\
& \times\left[\phi_{k}^{*}\left(\mathbf{a}_{\mu}\right) \nabla \phi_{l}^{*}\left(\mathbf{a}_{\mu}\right)-\phi_{l}^{*}\left(\mathbf{a}_{\mu}\right) \nabla \phi_{k}^{*}\left(\mathbf{a}_{\mu}\right)\right]
\end{aligned}
$$

is the curvature of the triplet pair density at the position $\mathbf{a}_{\mu}$. Those parts of $g^{(t)}\left(\mathbf{a}_{\mu}\right)$ which are connected with the samespin matrices $P_{i j, k l}^{\sigma \sigma}$ are the Fermi hole curvatures:

$$
\begin{aligned}
g^{\sigma}\left(\mathbf{a}_{\mu}\right)= & \sum_{i<j}^{\sigma} \sum_{k<l}^{\sigma} P_{i j, k l}^{\sigma \sigma} \\
& \times\left[\phi_{i}\left(\mathbf{a}_{\mu}\right) \nabla \phi_{j}\left(\mathbf{a}_{\mu}\right)-\phi_{j}\left(\mathbf{a}_{\mu}\right) \nabla \phi_{i}\left(\mathbf{a}_{\mu}\right)\right] \\
& \times\left[\phi_{k}^{*}\left(\mathbf{a}_{\mu}\right) \nabla \phi_{l}^{*}\left(\mathbf{a}_{\mu}\right)-\phi_{l}^{*}\left(\mathbf{a}_{\mu}\right) \nabla \phi_{k}^{*}\left(\mathbf{a}_{\mu}\right)\right] .
\end{aligned}
$$

Thus, the same-spin electron pairs have the following contribution to the triplet pair population in the micro-cell $\mu$ :

$D_{\mu}^{\alpha \alpha}+D_{\mu}^{\beta \beta} \approx \frac{1}{12} V_{\mu}^{8 / 3}\left[g^{\alpha}\left(\mathbf{a}_{\mu}\right)+g^{\beta}\left(\mathbf{a}_{\mu}\right)\right]$.

The remaining part of $D_{\mu}^{(t)}$ accounts for the contribution of opposite-spin electron pairs (this should not be mistaken for the number of opposite-spin pairs in micro-cell $\mu$, which is proportional to the on-top pair density, cf. Part II).

The volume $V_{\mu}$ of the micro-cell $\mu$ enclosing $D_{\mu}^{(t)}$ triplet pairs is approximately given by (cf. Eq. 22):

$V_{\mu} \approx\left[D_{\mu}^{(t)}\right]^{3 / 8}\left[\frac{12}{g^{(t)}\left(\mathbf{a}_{\mu}\right)}\right]^{3 / 8}$.

With the restriction $D^{(t)}$ (without the index $\mu$ because the restriction applies to all micro-cells) the integration of the 
triplet density $\rho^{(t)}$ over the corresponding $\omega$-restricted partitioning yields the $\omega$-restricted distribution of charges $\zeta_{D}^{(t)}$ (the superscript indicates that the triplet charge is sampled in the micro-cells):

$$
\begin{aligned}
\zeta_{D}^{(t)}\left(\mathbf{a}_{\mu}\right) & \approx \rho^{(t)}\left(\mathbf{a}_{\mu}\right) V_{\mu} \\
& \approx\left[D^{(t)}\right]^{3 / 8} \rho^{(t)}\left(\mathbf{a}_{\mu}\right)\left[\frac{12}{g^{(t)}\left(\mathbf{a}_{\mu}\right)}\right]^{3 / 8} .
\end{aligned}
$$

For fixed infinitesimally small $D^{(t)}$ we can define ELI for triplet-coupled electrons $\Upsilon_{D}^{(t)}$ (the triplet charge in the microcells is sampled):

$\Upsilon_{D}^{(t)}(\mathbf{r})=\rho^{(t)}(\mathbf{r}) \tilde{V}_{D^{(t)}}(\mathbf{r})$,

with the pair-volume function $\tilde{V}_{D^{(t)}}(\mathbf{r})$ for the tripled pairs:

$$
\tilde{V}_{D^{(t)}}(\mathbf{r})=\left[\frac{12}{g^{(t)}(\mathbf{r})}\right]^{3 / 8} .
$$

Observe the change from the discrete position representation $\mathbf{a}_{\mu}$ to the quasi-continuous representation $\mathbf{r}$. Following Eq. $28 \mathrm{ELI}$ is proportional to the triplet charge needed to form the fixed fraction $D^{(t)}$ of a triplet pair. Of course, only if the restriction $D^{(t)}$ is explicitly given, the corresponding $\omega$-restricted space partitioning could be determined, i.e., the number of the micro-cells and their discrete positions $\mathbf{a}_{\mu}$. Without the explicite given value of $D^{(t)}$ we only know that the number of micro-cells is some finite number. Nevertheless, the ELI value $\Upsilon_{D}^{(t)}(\mathbf{r})$ can be computed at any position $\mathbf{r}$, bearing in mind that it is always possible to find such restriction $D^{(t)}$ that the chosen positions will be hit. Thus, the distribution of ELI values can be made dense to any extent, however, not continuous. We use the term quasi-continuous for such distribution of values.

\subsection{ELI for singlet-coupled electrons}

Using the approach of $q$-restricted space partitioning, as decribed in Part II for ELIA, let as divide the whole space into compact space filling mutually exclusive micro-cells enclosing a fixed amount of singlet charge $q^{(s)}$. The volume $V_{\mu}$ of the micro-cell $\mu$ centred around the position $\mathbf{a}_{\mu}$ of such $q^{(s)}$-restricted space partitioning is then approximately given by:

$V_{\mu} \approx q^{(s)} \frac{1}{\rho^{(s)}\left(\mathbf{a}_{\mu}\right)}$.

The integration of the singlet pair density $\rho_{2}^{(s)}$ over the microcells yields the $q^{(s)}$-restricted distribution of singlet pair populations $\zeta_{q}^{(s)}$, which is approximately given by the on-top value of the singlet pair density $\rho_{2}^{(s)}\left(\mathbf{a}_{\mu}, \mathbf{a}_{\mu}\right)$ multiplied by the squared micro-cell volume $V_{\mu}^{2}$ (cf. Part II):

$\zeta_{q}^{(s)}\left(\mathbf{a}_{\mu}\right) \approx V_{\mu}^{2} \quad \rho_{2}^{(s)}\left(\mathbf{a}_{\mu}, \mathbf{a}_{\mu}\right) \approx\left[q^{(s)}\right]^{2} \frac{\rho_{2}^{(s)}\left(\mathbf{a}_{\mu}, \mathbf{a}_{\mu}\right)}{\left[\rho^{(s)}\left(\mathbf{a}_{\mu}\right)\right]^{2}}$.

The on-top singlet pair density is the diagonal part of the singlet 2-matrix, Eq. 16, which for $\mathbf{r}_{1}=\mathbf{r}_{2}=\mathbf{a}_{\mu}$ reduces to:

$\rho_{2}^{(s)}\left(\mathbf{a}_{\mu}, \mathbf{a}_{\mu}\right)=\sum_{i, k}^{n} \sum_{j, l}^{n} P_{i j, k l}^{\alpha \beta} \phi_{i}\left(\mathbf{a}_{\mu}\right) \phi_{j}\left(\mathbf{a}_{\mu}\right) \phi_{k}^{*}\left(\mathbf{a}_{\mu}\right) \phi_{l}^{*}\left(\mathbf{a}_{\mu}\right)$.

For infinitesimally small restriction $q^{(s)}$ we can define ELI for the singlet-coupled electrons $\Upsilon_{q}^{(s)}$ :

$\Upsilon_{q}^{(s)}(\mathbf{r})=\frac{1}{2}\left[\frac{N+2}{N-1}\right]^{2} \frac{\rho_{2}^{(s)}(\mathbf{r}, \mathbf{r})}{\left[\rho^{(s)}(\mathbf{r})\right]^{2}}$,

which reproduces in the spin-unpolarized case the ELIA functional for the opposite-spin pairs (cf. Part II, where the charge product $q_{\alpha} q_{\beta}$ was used as the restriction). The $\mathrm{N}$-dependent factor in the expression for singlet pair ELI ensures that $\Upsilon_{q}^{(s)}(\mathbf{r})=1$ for closed shell Hartree-Fock wavefunction (in which case the on-top singlet pair density, Eq. 32, yields $\left.\rho(\mathbf{r})^{2} / 4\right)$.

\section{Results}

Deeper insight into the relationship between ELI based on same-spin pair population on the one hand and the triplet pair population on the other can be gained from the examination of the opposite-spin contributions originating from wavefunctions given either by a single determinant or by many determinants. For single-determinantal wavefunction the corresponding 2-matrix is much more easy to survey.

\subsection{Single-determinantal wavefunctions}

The Hartree-Fock (HF) calculation involves only one configuration. If only a single Slater determinant is used, then, assuming real-valued orbitals, the curvature of the triplet pair density, Eq. 23, simplifies to:

$g^{(t)}\left(\mathbf{a}_{\mu}\right)=\sum_{i<j}^{n} P_{i j, i j}^{(t)}\left[\phi_{i}\left(\mathbf{a}_{\mu}\right) \nabla \phi_{j}\left(\mathbf{a}_{\mu}\right)-\phi_{j}\left(\mathbf{a}_{\mu}\right) \nabla \phi_{i}\left(\mathbf{a}_{\mu}\right)\right]^{2}$

In this case, the contribution of the same-spin terms to the triplet pair population, Eq. 25, can be written as (utilizing the 
relation $g^{\sigma}=2 \rho_{\sigma} \tau_{\sigma}-1 / 4\left(\nabla \rho_{\sigma}\right)^{2}$, cf. Part I):

$D_{\mu}^{\alpha \alpha}+D_{\mu}^{\beta \beta} \approx$

$\frac{1}{6} V_{\mu}^{8 / 3}\left[\rho_{\alpha} \tau_{\alpha}-\frac{1}{8}\left(\nabla \rho_{\alpha}\right)^{2}+\rho_{\beta} \tau_{\beta}-\frac{1}{8}\left(\nabla \rho_{\beta}\right)^{2}\right]$,

with $\tau_{\sigma}=\frac{1}{2} \sum_{i}^{\sigma}\left|\nabla \phi_{i}\right|^{2}$. Similarly, the remaining oppositespin contribution to the triplet pair population reads:

$\frac{1}{12} V_{\mu}^{8 / 3}\left[\rho_{\alpha} \tau_{\beta}+\rho_{\beta} \tau_{\alpha}-\frac{1}{4} \nabla \rho_{\alpha} \nabla \rho_{\beta}\right]$.

Apparently, for single-determinantal wavefunctions the triplet pair population in the micro-cell $\mu$ can be written as:

$$
\begin{aligned}
& D_{\mu}^{(t)} \approx \frac{1}{6} V_{\mu}^{8 / 3} \\
& \quad \times\left[\rho \tau-\frac{1}{8}(\nabla \rho)^{2}-\frac{\rho_{\alpha} \tau_{\beta}+\rho_{\beta} \tau_{\alpha}}{2}+\frac{1}{8} \nabla \rho_{\alpha} \nabla \rho_{\beta}\right] .
\end{aligned}
$$

In closed-shell case the above equation simplifies to:

$D_{\mu}^{(t)} \approx \frac{1}{8} V_{\mu}^{8 / 3}\left[\rho \tau-\frac{1}{8}(\nabla \rho)^{2}\right]$.

If the micro-cells are determined by the $q$-restricted partitioning, i.e., each micro-cell contains the same total charge $q$, the volumes of the micro-cells can be expressed according to $V_{\mu}=q / \rho\left(\mathbf{a}_{\mu}\right)$, cf. Part I. For infinitesimally small $q$ Eq. 37 yields the $q$-restricted triplet pair population for the HF case:

$$
\begin{aligned}
\zeta_{q, H F}^{(t)} \approx & q^{8 / 3} \frac{1}{6} \frac{\rho \tau-\frac{1}{8}(\nabla \rho)^{2}}{\rho^{8 / 3}} \\
& -q^{8 / 3} \frac{1}{6} \frac{\frac{1}{2}\left(\rho_{\alpha} \tau_{\beta}+\rho_{\beta} \tau_{\alpha}\right)-\frac{1}{8} \nabla \rho_{\alpha} \nabla \rho_{\beta}}{\rho^{8 / 3}} .
\end{aligned}
$$

Only the first term of the above equation is proportional to the kernel of the "closed-shell" ELF formula from Ref. [6] (however, in ELI for triplet coupled electrons $\Upsilon_{D}^{(t)}$ the triplet density is sampled, which only in closed shell case is proportional to the total density).

In case of closed-shell $(S=0)$ single-determinantal wavefunctions the elements of the triplet 2-matrix, cf. Eq. 21, reduce to:

$P_{i j, k l}^{(t)}=3 P_{i j, k l}^{\alpha \alpha}$.

In this case, the triplet pair-volume function $\tilde{V}_{D^{(t)}}$, Eq. 29 , is connected with the pair-volume function for the same-spin pairs $\tilde{V}_{D^{\alpha \alpha}}$ :

$\tilde{V}_{D^{(t)}}=\left[\frac{12}{g^{(t)}}\right]^{3 / 8}=\left[\frac{12}{3 g^{\alpha}}\right]^{3 / 8}=\left(\frac{1}{3}\right)^{3 / 8} \tilde{V}_{D^{\alpha \alpha}}$.

At the same time (cf. Eq. 5 with $\rho=2 \rho_{\alpha}$ ):

$\rho^{(t)}=\frac{3}{2} \frac{N-2}{N-1} \rho_{\alpha}$.

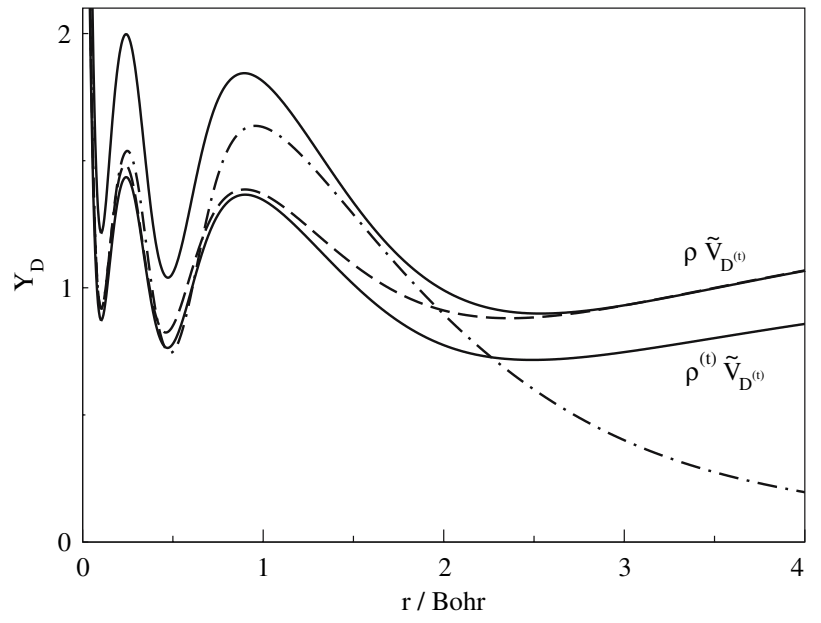

Fig. 1 ELI for the $\mathrm{Cr}\left({ }^{7} \mathrm{~S}\right)$ atom (HF calculation). Dashed line majority spin; dash-dotted line minority spin; lower solid line sampling of triplet density; upper solid line sampling of total density

Then, in closed-shell case, ELI for triplet coupled electrons is proportional to ELI for same-spin pairs $\Upsilon_{D}^{\alpha}=\rho_{\alpha} \tilde{V}_{D^{\alpha \alpha}}$ :

$\Upsilon_{D}^{(t)}=\rho^{(t)} \tilde{V}_{D^{(t)}}=\frac{3^{5 / 8}}{2} \frac{N-2}{N-1} \Upsilon_{D}^{\alpha}$

Of course, in the spin-polarized case the ELI for the majority spin differs from the one for the minority spin as can be seen in Fig. 1 for the $\mathrm{Cr}$ atom (ELI was computed with the program DGrid [15] using the basis set of Clementi and Roetti [16]). However, in the first two shells, the ELI values for the single spin are similar and very close to the ELI for the triplet coupled electrons (lower solid line). Sampling the total density in the micro-cells, i.e., computing $\rho \tilde{V}_{D^{(t)}}$ yields the upper solid line in Fig. 1. In this case the resulting values in the first two shells are roughly $2 / 3^{3 / 8}$ times higher than $\Upsilon_{D}^{\alpha}$, whereas being almost identical with $\Upsilon_{D}^{\alpha}$ in the valence shell (where the majority-spin density almost recovers the total density).

Silvi [17] proposed to use for spin-polarized systems a "modified ELF" formula. The kernel $\chi_{\bmod }$ of this "modified ELF" includes both same-spin components (using the conditional pair probability densities $P_{\text {cond }}^{\alpha \alpha}$ and $P_{\text {cond }}^{\beta \beta}$, cf. Eq. 30 in Ref. [17]):

$\chi_{\text {mod }}=\frac{1}{3} \frac{\rho_{\alpha} \nabla^{2} P_{\text {cond }}^{\alpha \alpha}+\rho_{\beta} \nabla^{2} P_{\text {cond }}^{\beta \beta}}{\rho^{8 / 3}}$.

With the relationship $\rho_{\alpha} \nabla^{2} P_{\text {cond }}^{\alpha \alpha}=g^{\alpha \alpha}$ it can be seen that the kernel $\chi_{\text {mod }}$ is actually proportional to the same-spin part of the triplet pair population given by Eq. 25 (replacing the micro-cell volume $V_{\mu}$ by $1 / \rho$, i.e., using $q$-restricted space partitioning):

$\chi_{\bmod }=\frac{1}{3} \frac{1}{\rho^{8 / 3}}\left(g^{\alpha}+g^{\beta}\right)$. 


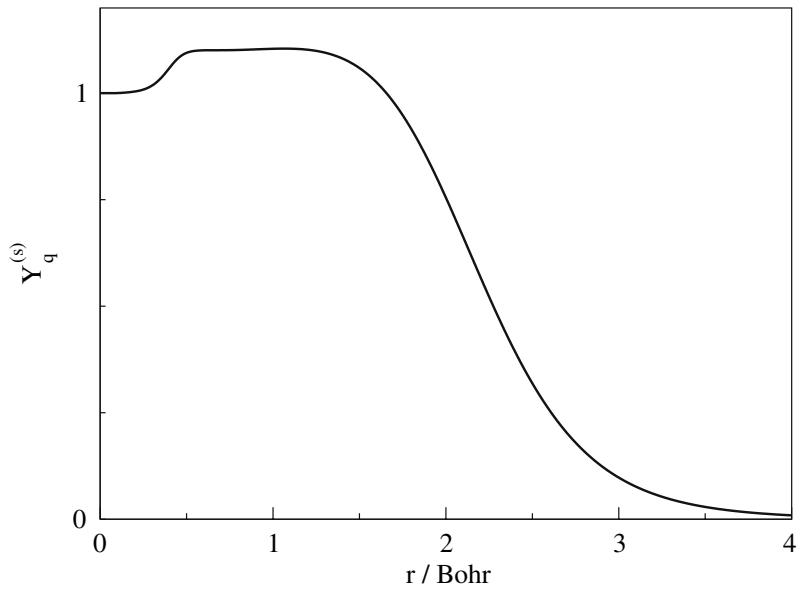

Fig. $2 \mathrm{Cr}\left({ }^{7} \mathrm{~S}\right)$ atom (HF calculation): ELI for singlet coupled electrons

For closed-shell HF wavefunction Silvi's expression would be proportional to the triplet pair population, cf. Eq. 38 (and to the same-spin population as well). However, for spinpolarized systems the opposite-spin term in the triplet pair population will in general differ from the same-spin term. Correspondingly, also the kernel of ELI for triplet-coupled electrons will differ from the kernel of the "modified ELF" formula.

For single-determinantal wavefunctions the on-top pair density is given by the product $\rho_{\alpha} \rho_{\beta}$. Fixing the charge product $q_{\alpha} q_{\beta}$ for the $\omega$-restricted partitioning (cf. ELIA in Part II) yields a uniform opposite-spin pair population over the micro-cells of such partitioning. In this case, a non-uniform opposite-spin pair population can emerge only from multideterminantal wavefunctions. However, using a fixed amount of singlet electrons as the restriction for the space partitioning, as suggested in previous section for $\Upsilon_{q}^{(s)}$, can change the situation completely. Now, in spin-polarized case even for single-determinantal wavefunction the singlet pair population over the micro-cells becomes non-uniform, cf. Fig. 2 for the $\mathrm{Cr}$ atom, because the squared singlet density $\rho^{(s)}$ is no more proportional to the product $\rho_{\alpha} \rho_{\beta}$ (which is true for $\rho_{s}=0$, cf. Eq. 5).

\subsection{Spin-adapted configurations}

For singlet wavefunction ( $S=0$ ), an open-shell restricted determinant is not eigenfunction of the total spin operator. Instead, an appropriate linear combination of determinants must be taken (spin-adapted configuration). As an example, let us consider the excited singlet state of the minimal basis $\mathrm{H}_{2}$ molecule, described by determinants with the $\sigma_{g}$ and $\sigma_{u}$ orbitals occupied. The electrons must have opposite spin which leads to two determinants:

$$
\begin{aligned}
& \frac{1}{\sqrt{2}}\left[\sigma_{g}\left(\mathbf{r}_{1}\right) \alpha(1) \sigma_{u}\left(\mathbf{r}_{2}\right) \beta(2)-\sigma_{u}\left(\mathbf{r}_{1}\right) \beta(1) \sigma_{g}\left(\mathbf{r}_{2}\right) \alpha(2)\right] \\
& \frac{1}{\sqrt{2}}\left[\sigma_{u}\left(\mathbf{r}_{1}\right) \alpha(1) \sigma_{g}\left(\mathbf{r}_{2}\right) \beta(2)-\sigma_{g}\left(\mathbf{r}_{1}\right) \beta(1) \sigma_{u}\left(\mathbf{r}_{2}\right) \alpha(2)\right]
\end{aligned}
$$

The singlet spin-adapted configuration ${ }^{1} \Psi$ is the sum of the above determinants [18]:

$$
\begin{aligned}
{ }^{1} \Psi\left(\mathbf{r}_{1}, \mathbf{r}_{2}\right)= & \frac{1}{2}\left[\sigma_{g}\left(\mathbf{r}_{1}\right) \sigma_{u}\left(\mathbf{r}_{2}\right)+\sigma_{u}\left(\mathbf{r}_{1}\right) \sigma_{g}\left(\mathbf{r}_{2}\right)\right] \\
& \times[\alpha(1) \beta(2)-\beta(1) \alpha(2)]
\end{aligned}
$$

The contribution of a particular determinant to the spinadapted configuration is given by the symmetry. Unlike the CI procedure, there is no optimization of the determinant weight. The optimization of the spin-adapted configuration concerns only the orbitals themselves. From the above singlet spin-adapted configuration ${ }^{1} \Psi$ the electron pair density $\rho_{2}$ can be computed:

$\rho_{2}\left(\mathbf{r}_{1}^{\prime} \mathbf{r}_{2}^{\prime}, \mathbf{r}_{1} \mathbf{r}_{2}\right)=\int \mathrm{d} \sigma_{1} \int \Psi\left(\mathbf{x}_{1}^{\prime} \mathbf{x}_{2}^{\prime}\right) \Psi^{*}\left(\mathbf{x}_{1} \mathbf{x}_{2}\right) \mathrm{d} \sigma_{2}$,

yielding

$\rho_{2}\left(\mathbf{r}_{1}^{\prime} \mathbf{r}_{2}^{\prime}, \mathbf{r}_{1} \mathbf{r}_{2}\right)=\frac{1}{2}\left\|\sigma_{g}\left(\mathbf{r}_{1}^{\prime}\right) \sigma_{u}\left(\mathbf{r}_{2}^{\prime}\right)\right\|\left\|\sigma_{g}^{*}\left(\mathbf{r}_{1}\right) \sigma_{u}^{*}\left(\mathbf{r}_{2}\right)\right\|$.

In this simple case the exchange of the unprimed coordinates, cf. Eq. 3, has no effect on the above 2-matrix (matrix permanents are used). Thus, the 2-matrix in Eq. 49 directly yields the singlet 2-matrix $\rho_{2}^{(s)}$ (of course, the triplet 2-matrix vanishes). At the same time, the singlet 2-matrix $\rho_{2}^{(s)}$ is twice the opposite-spin 2-matrix $\rho_{2}^{\alpha \beta}$.

In our example $S=0$ and $N=2$, i.e., $\rho^{(s)}=\rho$ (with the total electron density $\rho=\sigma_{g}^{2}+\sigma_{u}^{2}$ ). Let us perform $q^{(s)}$-restricted partitioning of the space, i.e., each micro-cell $\mu$ with the volume $V_{\mu}$ encloses the same fixed amount of singlet charge $q^{(s)} \approx \rho^{(s)} V_{\mu}$. The singlet pair population in micro-cell $\mu$ is approximately given by the product of the squared micro-cell volume $V_{\mu}^{2}$ and the on-top value of the singlet pair density, cf. Part II. By inspection of Eq. 49 (setting all coordinates to the same value) this gives the singlet pair population $\zeta_{q, s a}^{(s)}$ for the spin-adapted configuration:

$\zeta_{q, s a}^{(s)} \approx V_{i}^{2} 2 \sigma_{g}^{2} \sigma_{u}^{2} \approx 2 q^{2} \frac{\sigma_{g}^{2} \sigma_{u}^{2}}{\left[\sigma_{g}^{2}+\sigma_{u}^{2}\right]^{2}}$.

From this it follows that the localizability indicator for singlet-coupled electrons described by spin-adapted configuration is nonuniform. Thus, already the symmetry restriction introduces certain correlation of motion of opposite-spin electrons. The difference of the two determinants in Eq. 46 yields the triplet spin-adapted configuration with $S=1$ and the spin projection $M_{S}=0$. The determinants with the spin 
projection $M_{S}= \pm 1$ have both $\sigma_{g}$ and $\sigma_{u}$ occupied by electrons with the same spin. The linear combination of the three determinants describes the triplet wavefunction ${ }^{3} \Psi$. The electron pair density $\rho_{2}$ derived from ${ }^{3} \Psi$ reads:

$\rho_{2}\left(\mathbf{r}_{1}^{\prime} \mathbf{r}_{2}^{\prime}, \mathbf{r}_{1} \mathbf{r}_{2}\right)=\frac{1}{2}\left|\sigma_{g}\left(\mathbf{r}_{1}^{\prime}\right) \sigma_{u}\left(\mathbf{r}_{2}^{\prime}\right)\right|\left|\sigma_{g}^{*}\left(\mathbf{r}_{1}\right) \sigma_{u}^{*}\left(\mathbf{r}_{2}\right)\right|$.

The above 2-matrix directly yields the triplet 2-matrix (now the singlet 2-matrix vanishes). The ELI for the triplet-coupled electrons can be computed according to Eqs. 28, 29 and 23. Of course, in this simple case the ELI for triplet pairs is proportional to the ELI for same-spin pairs, because both same-spin and opposite-spin pair densities are given by identical spatial function $\left[\sigma_{g}\left(\mathbf{r}_{1}\right) \sigma_{u}\left(\mathbf{r}_{2}\right)-\sigma_{u}\left(\mathbf{r}_{1}\right) \sigma_{g}\left(\mathbf{r}_{2}\right)\right]^{2}$.

\subsection{Multi-determinantal wavefunctions}

For correlated wavefunctions from CI calculation the simple expression using the electron densities and $\tau$, cf. Eq. 37, must be replaced by the orbital sum of Eq. 23, i.e., the full 2-matrix must be known to compute ELI for correlated wavefunctions. As an example for spin-polarized system the ground state ${ }^{3} \Sigma_{g}^{-}$of the oxygen molecule was computed. The complete active space calculation for 12 electrons in 12 orbitals was performed with Gaussian03 [19] using the cc-pVDZ basis set (bond distance $1.218 \AA$ [20]). The density matrices were created with the program DGrid and used to compute ELI for the majority and minority spin, respectively.

The corresponding diagrams are shown in Fig. 3. The ELI signatures of the lone pairs (the ring shaped gold colored localization domains) in the diagram for the majority spin, Fig. 3a, resemble the situation in the $\mathrm{F}_{2}$ molecule (cf. Part I). The red colored localization domain encloses the single ELI attractor at the bond midpoint. The ELI for minority spin, Fig. $3 b$, shows signatures very different from the previous one. Now each lone pair signature encloses an ELI attractor on the bond axis, resembling the lone pair situation in $\mathrm{N}_{2}$ molecule (cf. Part I). Interestingly, the minority ELI shows a "bifurcated" bond attractor (the two red colored localization domains), as was found for $\mathrm{F}_{2}$ (Part I). The two spin parts can be unified into single diagram using ELI for triplet coupled electrons, Fig. 3c. In this total picture the lone pairs are represented by ring shaped localization domains. The bond signature encloses a single ELI attractor at bond midpoint.

The ELI for singlet coupled electrons, Fig. 4, shows distinct signatures for the lone pairs and the bond, similar to the ELI for triplet coupled electrons. The two ring shaped localization domains characterize the lone pairs as regions, where more singlet coupled pairs are formed from the fixed singlet charge, than in the the surrounding. The same is true for the bond signature described by the red colored localization domain (enclosing a single ELI attractor). In the regions of low singlet ELI values the opposite spin electrons are avoid-
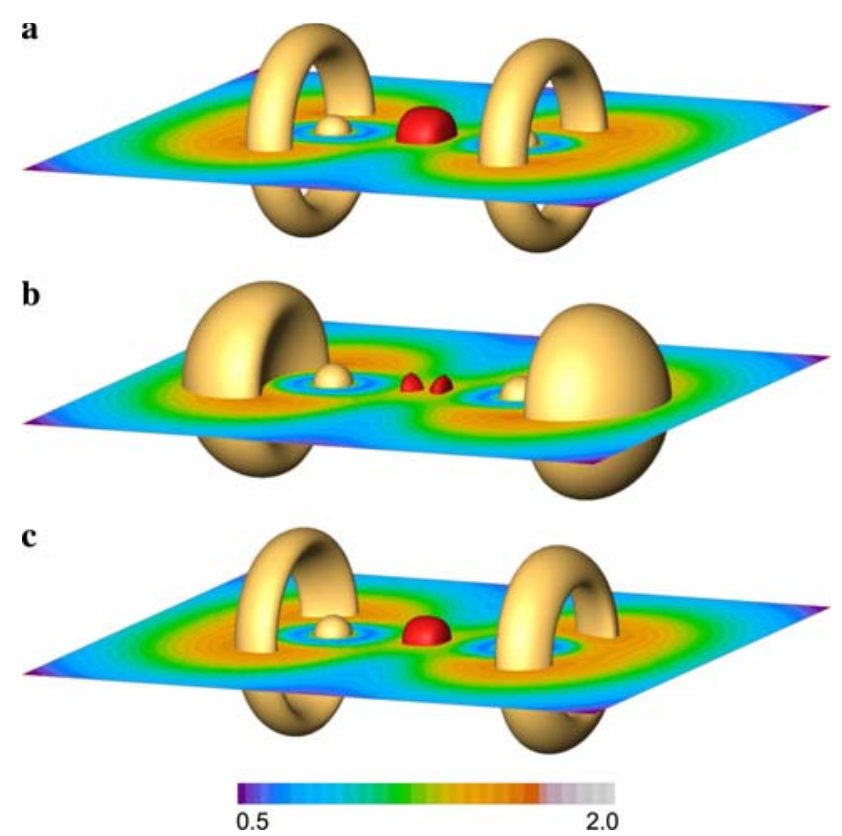

Fig. 3 ELI for the ground state ${ }^{3} \Sigma_{g}^{-}$of the $\mathrm{O}_{2}$ molecule. a 1.7 (gold) and 1.3-localization domains (red) for the majority spin. b 1.7 (gold) and 1.482-localization domains (red) for the minority spin. c 1.55 (gold) and 1.3-localization domains (red) for the triplet coupled electrons. The color map applies to the slices

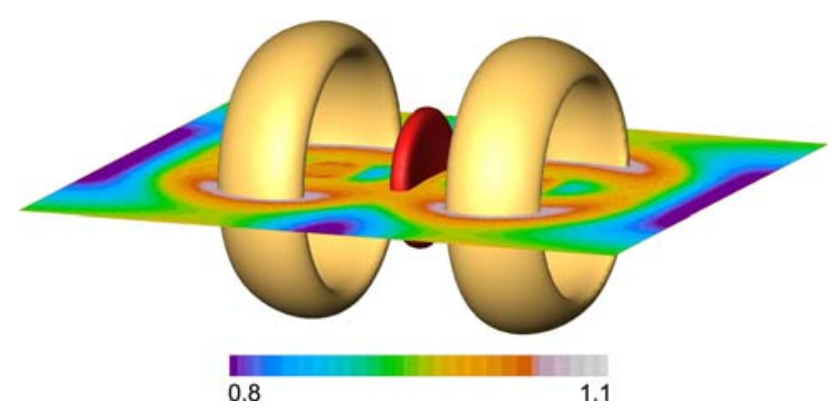

Fig. 4 ELI for singlet coupled electrons for the ground state ${ }^{3} \Sigma_{g}^{-}$of the $\mathrm{O}_{2}$ molecule. 1.05 (gold) and 1.0-localization domains (red). The color map applies to the slice

ing each other (thus forming less pairs from the fixed amount of charge).

ELI for triplet-coupled electrons is proportional to the triplet charge (the sampling quantity) that is needed to form fixed fraction of a triplet pair (the control quantity, determining the space partitioning). Instead of the triplet charge in the microcells of the $D^{(t)}$-restricted partitioning it is possible to utilize the total charge. This is fully consistent with the approach of restricted populations. Then ELI would be given by the expression:

$\Upsilon_{D^{(t)}}(\mathbf{r})=\rho(\mathbf{r}) \tilde{V}_{D^{(t)}}(\mathbf{r})=\rho(\mathbf{r})\left[\frac{12}{g^{(t)}(\mathbf{r})}\right]^{3 / 8}$.

In the absence of spin polarization, i.e., $\rho_{s}(\mathbf{r})=0$, the triplet density $\rho^{(t)}$ is a certain fraction of the total electron density, 
cf. Eq. 5. In this case $\Upsilon_{D^{(t)}}$ is proportional to $\Upsilon_{D}^{(t)}$ and their topology remains the same. However, for spin-polarized calculation, the value of ELI will be influenced by the spin density when comparing $\Upsilon_{D^{(t)}}$ and $\Upsilon_{D}^{(t)}$.

Similarly, ELI for singlet-coupled electrons could be based on $q$-restricted space partitioning, i.e., each micro-cell enclosing a fixed total charge. Then the expression for ELI reads:

$\Upsilon_{q}(\mathbf{r})=4 \frac{\rho_{2}^{(s)}(\mathbf{r}, \mathbf{r})}{[\rho(\mathbf{r})]^{2}}$

The factor 4 ensures $\Upsilon_{q}=1$ for closed-shell HF wavefunction. Like in the case of triplet-coupled electrons, only in the absence of spin polarization the ELI for the two restriction schemes (micro-cells enclosing fixed total charge, respectively fixed number of singlet electrons) are proportional to each other.

\section{Conclusions}

The electron pair density can be decomposed into the symmetric and antisymmetric parts. Such decomposition describes electrons that couple to a singlet and triplet pair, respectively. The division of the whole space into compact mutually exclusive space filling micro-cells enclosing a fixed fraction of a triplet pair defines the $D^{(t)}$-restricted space partitioning. The electron localizability indicator ELI for triplet coupled electrons is proportional to the triplet charges in the micro-cells of the $D^{(t)}$-restricted partitioning. It describes the correlation of electronic motion of electrons forming a triplet pair. In case of spin-polarized calculation this approach yields a single functional which takes into account both spin channels at once. Additionally, it was shown that the ELI for singlet-coupled electrons reproduces in the spin-unpolarized case the electron localizability indicator for antiparallel-spin pairs ELIA.

\section{References}

1. Kohout M, Pernal K, Wagner FR, Grin Yu (2004) Theor Chem Acc 112:453

2. Bader RFW, Stephens ME (1974) Chem Phys Lett 26:445
3. Kohout M, Pernal K, Wagner FR, Grin Yu (2005) Theor Chem Acc 113:287

4. Becke AD, Edgecombe KE (1990) J Chem Phys 92:5397

5. Savin A, Jepsen O, Flad J, Andersen OK, Preuss H, von Schnering HG (1992) Angew Chem Int Ed Engl 31:187

6. Kohout M, Savin A (1996) Int J Quantum Chem 60:875

7. Kutzelnigg W (1963) Z Naturforschg 18:1058

8. Kutzelnigg W, Mukherjee D (2002) J Chem Phys 116:4787

9. Löwdin P-O (1955) Phys Rev 97:1474

10. Parr RG, Yang W (1989) Density-functional theory of atoms and molecules. Oxford University Press, Oxford

11. Kutzelnigg W (2002) In: Rychlewski J (ed) Explicitly correlated wave functions in chemistry and physics: Theory and applications. Kluwer, The Netherlands, pp 14-17

12. Cooper DL, Ponec R, Thorsteinsson T (1994) Int J Quantum Chem 57:501

13. Kohout M (2007) Faraday Discuss 135:43

14. Wagner FR, Bezugly V, Kohout M, Grin Yu (2007) Chem Eur J 13:5724

15. Kohout M (2007) DGrid, Version 4.3

16. Clementi E, Roetti C (1974) At Data Nucl Data Tables 14:218

17. Silvi B (2003) J Phys Chem A 107:3081

18. Szabo A, Ostlund NS (1996) Modern quantum chemistry. Dover Publications, New York

19. Frisch MJ, Trucks GW, Schlegel HB, Scuseria GE, Robb MA, Cheeseman JR, Montgomery JA Jr., Vreven T, Kudin KN, Burant JC, Millam JM, Iyengar SS, Tomasi J, Barone V, Mennucci B, Cossi M, Scalmani G, Rega N, Petersson GA, Nakatsuji H, Hada M, Ehara M, Toyota K, Fukuda R, Hasegawa J, Ishida M, Nakajima T, Honda Y, Kitao O, Nakai H, Klene M, Li X, Knox JE, Hratchian HP, Cross JB, Bakken V, Adamo C, Jaramillo J, Gomperts R, Stratmann RE, Yazyev O, Austin AJ, Cammi R, Pomelli C, Ochterski JW, Ayala PY, Morokuma K, Voth GA, Salvador P, Dannenberg JJ, Zakrzewski VG, Dapprich S, Daniels AD, Strain MC, Farkas O, Malick DK, Rabuck AD, Raghavachari K, Foresman JB, Ortiz JV, Cui Q, Baboul AG, Clifford S, Cioslowski J, Stefanov BB, Liu G, Liashenko A, Piskorz P, Komaromi I, Martin RL, Fox DJ, Keith T, Al-Laham MA, Peng CY, Nanayakkara A, Challacombe M, Gill PMW, Johnson B, Chen W, Wong MW, Gonzalez C, Pople JA (2004) Gaussian 03, Revision C.02, Gaussian Inc., Wallingford

20. Peterson KA, Kendall RA, Dunning TH Jr. (1993) J Chem Phys 99:9790 